\title{
Reintervention for arch obstruction after stage 1 reconstruction does not adversely affect survival or outcome at Fontan completion
}

\author{
Jean A. Ballweg, MD, ${ }^{\mathrm{a}}$ Troy E. Dominguez, MD, ${ }^{\mathrm{a}}$ Sarah Tabbutt, MD, PhD, ${ }^{\mathrm{b}}$ Jonathan J. Rome, MD, ${ }^{\mathrm{a}}$ \\ J. William Gaynor, MD, ${ }^{\mathrm{a}}$ Susan C. Nicolson, MD, ${ }^{\mathrm{a}}$ Thomas L. Spray, MD, ${ }^{\mathrm{a}}$ and Chitra Ravishankar, $\mathrm{MD}^{\mathrm{a}}$
}

Objective: To determine the effect of reintervention for coarctation after stage 1 reconstruction for hypoplastic left heart syndrome and variants on survival, suitability for Fontan, and morbidity at Fontan.

Methods: A retrospective review of echocardiograms, catheterizations, hospital records of patients who underwent stage 1 reconstruction from January 2002 to May 2005, with a cross-sectional analysis of hospital survivors, was performed. Kaplan-Meier curves were derived for patients alive more than 30 days after stage 1 reconstruction.

Results: A total of 176 patients underwent stage 1 reconstruction. Forty-three patients $(23 \%)$ underwent balloon angioplasty $(n=43)$ or surgical intervention $(n=4)$ for re-coarctation. Median time to intervention was 123 (1-316) days. Seven of 43 patients (16\%) underwent more than 1 balloon angioplasty. Thirty-nine patients underwent intervention before stage 2 reconstruction, and 4 patients had intervention between stage 2 reconstruction and Fontan. Kaplan-Meier curves showed no difference in freedom from death or transplant between patients who did and did not undergo intervention for re-coarctation. Fontan completion was performed in 107 patients. By echocardiogram, the prevalence of moderate to severe ventricular dysfunction between groups was similar at Fontan; however, significant atrioventricular valve regurgitation was more common in patients who required intervention ( $28 / 33$ vs $40 / 65, P=.02)$. Overall Fontan mortality was $2 \%$ and not different between groups. Length of stay was not different between patients with and without re-coarctation.

Conclusions: Reintervention for coarctation after stage 1 reconstruction is common. Hemodynamic differences between groups did not affect Fontan completion, mortality, or hospital length of stay. Follow-up is necessary to determine the impact of re-coarctation on longer-term mortality and morbidity. (J Thorac Cardiovasc Surg 2010;140:545-9)

Multiple factors have resulted in improved survival among infants born with functional single-ventricle lesions. These factors include surgical modifications, improved surgical technique, modifications to preoperative and postoperative care, and a better understanding of the cardiac physiology of patients with single ventricles undergoing surgical palliation. ${ }^{1-6}$ Attention has now shifted toward understanding variables that may result in mortality or less favorable hemodynamic and functional status as the patients move through staged palliation. One such variable is the occurrence of recurrent arch obstruction. The reported incidence of recurrent arch obstruction after stage 1 reconstruction (S1R) ranges from $9 \%$ to $37 \% .^{7-14}$ Recurrent arch obstruction has been observed to result in worse ventricular systolic function after S1R for hypoplastic left

\footnotetext{
From the Children's Hospital of Philadelphia, ${ }^{\text {a }}$ Philadelphia, Pa; and University of California San Francisco, ${ }^{\mathrm{b}}$ San Francisco, Calif.

Disclosures: None.

Read at the Thirty-fifth Annual Meeting of the Western Thoracic Surgical Association, June 27, 2009, Banff, Alberta, Canada.

Received for publication June 19, 2009; revisions received May 13, 2010; accepted for publication June 6, 2010.

Address for reprints: Jean A. Ballweg, MD, Department of Pediatrics, Division of Pediatric Cardiology, The University of Tennessee, Le Bonheur Children's Hospital, 777 Washington Ave, Memphis, TN 38103 (E-mail: jballweg@uthsc.edu). $0022-5223 / \$ 36.00$

Copyright (C) 2010 by The American Association for Thoracic Surgery doi:10.1016/j.jtcvs.2010.06.013
}

heart syndrome (HLHS), with improvement toward previous baseline with timely intervention of the recurrent obstruction. ${ }^{15}$ In addition, a single postmortem study has demonstrated that recurrent arch obstruction was the primary cause of death in $14 \%$ of patients evaluated by autopsy after in-hospital death post-S1R. ${ }^{16}$ The aim of this study was to evaluate the consequences of re-coarctation after S1R or stage 2 reconstruction (S2R) on Fontan completion for HLHS and other single ventricle variants.

\section{MATERIALS AND METHODS \\ Study Patients and Design}

This study was a cross-sectional case series including all patients who underwent S1R for HLHS or variants at The Children's Hospital of Philadelphia between January 1, 2002, and May 1, $2005(n=176)$, with particular attention paid to patients alive more than 30 days after S1R $(n=156)$. Patient data were collected through April 16, 2009. This was a consent-waived study approved by The Children's Hospital of Philadelphia Institutional Review Board. Sources of information included the hospital medical records, the cardiac center and cardiac intensive care databases, and the records and reports provided by referring cardiologists. Recurrent arch obstruction was defined as those patients who underwent reintervention (surgical or balloon angioplasty) of the reconstructed aortic arch. Balloon angioplasties performed both at The Children's Hospital of Philadelphia and the referring institutions were included for analysis. There were no prospective criteria established for the diagnosis or treatment of recurrent arch obstruction. Blood pressure gradient was not used as a criterion for intervention because of the difficulty in accurate measurement, the possibility for small gradients in the presence of poor cardiac output, and the lack of a uniform number for intervention from institution 


\section{Abbreviations and Acronyms \\ HLHS = hypoplastic left heart syndrome \\ $\mathrm{S} 1 \mathrm{R}=$ stage 1 reconstruction \\ $\mathrm{S} 2 \mathrm{R}=$ stage 2 reconstruction}

to institution. The decision for intervention was made by the attending cardiologist and cardiac surgeon on the basis of echocardiographic evidence of recurrent arch obstruction: decreased ventricular systolic function, increased atrioventricular valve regurgitation or abnormal abdominal Doppler flow pattern, or catheterization data including pullback gradient and angiographic appearance.

We reviewed the most recent echocardiographic report before arch intervention for the patients with documented arch obstruction for assessment of ventricular systolic function and atrioventricular valve regurgitation. Postintervention echocardiographic data were recorded when available. These data were also recorded from echocardiographic reports before Fontan completion for all patients.

Hemodynamic data obtained at cardiac catheterization with conscious sedation or a general anesthetic at the time of arch intervention included pre- and post-balloon angioplasty gradients, ventricular end-diastolic pressure, common atrial pressure, and mean pulmonary artery pressure. These data were recorded at the time of pre-Fontan catheterization for patients who underwent repeat catheterization before Fontan completion. For patients without catheterization, upper- and lower-extremity blood pressure measurements were documented before Fontan completion.

\section{Statistical Analysis}

Analyses were performed for all patients who underwent S1R and for those who survived past 30 days of life.

\section{Descriptive Statistics}

Continuous variables are presented as mean \pm standard deviation for normally distributed data and median (range) otherwise. Categoric data and ordinal data are presented as $\mathrm{n}(\%)$.

\section{Patients With Recurrent Arch Obstruction}

Depending on the distribution, a paired analysis using the Wilcoxon signed-rank test or Student $t$ test was performed to compare hemodynamic data before and after balloon dilation angioplasty. The time to re-coarctation was studied using the Kaplan-Meier method to estimate re-coarctation rates at different time points and survival curves. Potential risk factors for recurrent arch obstruction were assessed using Cox proportional hazards regression.

\section{Comparisons Between Patients With and Without Arch Obstruction at the Time of Fontan}

The Student $t$ test or Wilcoxon rank-sum test was used to compare hemodynamic and physiologic data between patient groups depending on the distribution. For comparisons using binary or categoric data, a chi-square test or Fisher's exact test was used. The Wilcoxon rank-sum test was used to compare ordinal data, such as the degree of atrioventricular valve regurgitation, between groups.

\section{Survival Analysis}

The Kaplan-Meier method was used to estimate survival to death or transplant at different time points. Survival differences between those patients with and without arch obstruction were compared using the logrank test. The statistical analysis was performed using STATA 9.0 (Stata Corp, College Station, Tex).

\section{RESULTS}

Between January 1, 2002, and May 1, 2005, 176 patients underwent S1R for HLHS or single ventricle variants at The Children's Hospital of Philadelphia. Patients dying after surgery but before 30 days of life $(n=20)$ were excluded from the secondary analysis because they did not have the potential to become eligible for Fontan completion. Of the 156 patients included, $94(60 \%)$ were male and $62(40 \%)$ were female. Other patient characteristics, including cardiac anatomy, weight, and age at the time of S1R, are summarized in Table 1.

The S1R was performed by 4 surgeons, and all procedures used deep hypothermic circulatory arrest. The standard approach, independent of the type of pulmonary shunt used, included an atrial septectomy and division of the main pulmonary artery with side-to-side anastomosis to the diminutive ascending aorta without aortic transection. Routinely, the aorta was incised and the incision was carried across the arch of the aorta beyond the ductal insertion site onto the descending thoracic aorta. Ductal tissue was excised, and the proximal ascending aorta was tacked side to side to the pulmonary artery. The entire arch of the aorta was then augmented with a patch of pulmonary homograft. Co-artectomy was not routinely performed. Other patient and surgical characteristics have been summarized. ${ }^{17-19}$

\section{Patients With Recurrent Arch Obstruction}

The arch obstruction recurrence rate was 23\% (43/176) for the original patient cohort. Recurrent arch obstruction for patients alive at more than 30 days of life was $26 \%$ (42/156). Forty-three patients underwent balloon angioplasty or surgical intervention $(n=4)$. Three of the 4 patients requiring surgical intervention had supraaortic obstruction that was intervened upon at the time of the S2R. Seven patients $(16 \%)$ required more than 1 balloon angioplasty. Thirty-nine patients (91\%) had balloon angioplasty only between S1R and S2R, and 4 patients required balloon angioplasty for recurrent arch obstruction both before S2R and Fontan. The median time to surgical or catheter intervention was 123 (1-316) days.

The cardiac catheterization data at the time of intervention are as follows for those patients with recurrent arch obstruction. The gradient by pull-back measurement before intervention was $28.5(2-53) \mathrm{mm} \mathrm{Hg}$. After balloon angioplasty, the gradient decreased to $1(0-20) \mathrm{mm} \mathrm{Hg}$. The end-diastolic pressure in the ventricle at the time of arch obstruction was $10(4-15) \mathrm{mm} \mathrm{Hg}$, with a filling pressure of $7(3-15) \mathrm{mm}$ $\mathrm{Hg}$ and mean pulmonary artery pressure of $12(6-26) \mathrm{mm} \mathrm{Hg}$.

\section{Comparison of Patients With and Without Recurrent Arch Obstruction}

Among preoperative risk factors, the presence of significant noncardiac congenital anomalies was associated with recurrent arch obstruction $(P=.002)$. Other factors, such as birth weight less than $2.5 \mathrm{~kg}$, preoperative shock or 
TABLE 1. Patient characteristics at stage 1 reconstruction $(n=156)$

\begin{tabular}{lc}
\hline Anatomic subtype & \\
HLHS & $106(68 \%)$ \\
DORV or VSD with MA/MS & $20(13 \%)$ \\
Single LV & $16(10 \%)$ \\
UAVC & $10(6.5 \%)$ \\
Other & $4(2.5 \%)$ \\
Gender & \\
Male & $94(60 \%)$ \\
Female & $62(40 \%)$ \\
Weight (kg) & $3.1(1.4-4.5$ \\
Age at surgery (d) & $3(0-29)$ \\
\hline
\end{tabular}

$\overline{H L H S \text {, Hypoplastic left heart syndrome; DORV, double outlet right ventricle; VSD, }}$ ventricular septal defect; $M A / M S$, mitral atresia/mitral stenosis; $L V$, left ventricle; $U A V C$, unbalance atrioventricular canal.

presence of a genetic syndrome, or diagnosis of aortic atresia, were not associated with recurrent arch obstruction.

Survival. Of the 156 patients who survived more than 30 days of life, an additional 10 died before hospital discharge, 5 have undergone heart transplantation, 25 have been lost to interstage death, 1 has undergone conversion to a 2-ventricle palliation, and 8 await further palliative surgery. A total of 107 patients have undergone Fontan completion. Seven patients with previous balloon angioplasty for recurrent arch obstruction have died: 6 before Fontan completion and 1 after Fontan completion. Recurrent arch obstruction after balloon angioplasty was not documented by echocardiogram for any of the 7 deaths. Incidence of interstage death was not different between the 2 groups (arch obstruction $7 / 43$ [16\%] vs others 25/156 [16\%], $P=.842)$.

Patient and operative characteristics have been described for those who previously underwent Fontan completion. ${ }^{18}$ Patient weight at time of Fontan completion was not different between the 2 groups (arch obstruction $13 \pm 2 \mathrm{~kg}$ vs others $13 \pm 1.7 \mathrm{~kg}, P=.337$ ). There was no difference in patient age at time of Fontan completion between groups (arch obstruction 33.6 months [19.4-44.7] vs others 34.8 months [18.3-53.8], $P=.675$ ).

Cardiac catheterization findings at time of Fontan. Eighty-one of the 107 patients with Fontan completion underwent cardiac catheterization before surgery. Thirty of the patients $(81 \%)$ with previous recurrent arch obstruction underwent repeat cardiac catheterization before Fontan completion. Common atrial pressure was significantly higher at pre-Fontan catheterization for those patients with previous arch obstruction $(8$ [5-12] mm Hg vs others 6 [3-15] mm $\mathrm{Hg} ; P=.035)$. Mean pulmonary artery pressure was not different between the 2 groups, with a mean pulmonary artery pressure of 12 (9-26) $\mathrm{mm} \mathrm{Hg}$ in patients with previous arch obstruction versus $11(8-24) \mathrm{mm} \mathrm{Hg}$ in patients without obstruction $(P=.328)$. Pull-back gradient across the aortic arch or upper- and lower-extremity blood pressure measurement were available for 83 of the 107 patients who underwent Fontan completion. Notably, there was no difference in resid-

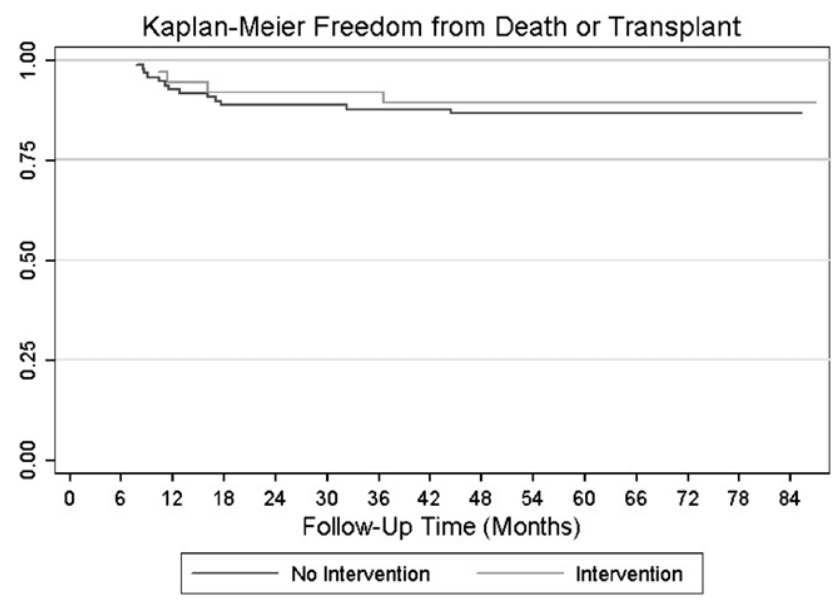

FIGURE 1. Kaplan-Meier: Freedom from death or transplant in patients with and without recurrent arch obstruction requiring reintervention before Fontan completion.

ual gradient from ascending to descending aorta by these measurement techniques between the 2 groups (arch obstruction 0 [0-10] $\mathrm{mm} \mathrm{Hg}$ vs no arch obstruction 0 [0-6] $\mathrm{mm} \mathrm{Hg}$; $P=0.355)$.

Echocardiogram findings at time of Fontan. Echocardiographic data were available for 98 patients before Fontan. There was no difference in the prevalence of qualitative moderate or severe ventricular systolic dysfunction between those with and without recurrent arch obstruction (2/33 $[6 \%]$ vs $4 / 65[6 \%], P=.86$ ). Qualitatively moderate or severe atrioventricular valve regurgitation was more common in patients with recurrent arch obstruction than in those without (28/33 [85\%] vs 40/65 [62\%], $P=.039)$.

Hospital length of stay, mortality, and late outcomes after Fontan. Hospital length of stay at the time of the Fontan completion was not different between those patients with recurrent arch obstruction (8.5 [2-73] days) and those without (8 [3-37] days, $P=.86$ ). Overall mortality after Fontan was $2 \%$. One patient with previous arch obstruction died of sepsis during the Fontan hospitalization. One patient without recurrent arch obstruction had a sudden cardiac arrest requiring extracorporeal membrane oxygenation cannulation with subsequent withdrawal of support secondary to embolic complications of heparin-induced thrombocytopenia.

The median duration of follow-up was 58 months (range, 1-87 months). There have been no deaths or transplants in either group after Fontan completion and hospital discharge through the current follow-up. By Kaplan-Meier analysis, recurrent arch obstruction did not influence survival or freedom from transplant at 5 years (arch obstruction 79\%, 95\% confidence limit, $63 \%-89 \%$, vs others $59 \%, 95 \%$ confidence limit, $59 \%-75 \% ; P=.24$; Figure 1).

\section{DISCUSSION}

This analysis of patients undergoing S1R between January 1, 2002, and May 1, 2005, demonstrates several significant 
findings. (1) Arch obstruction was common after S1R, as defined by need for intervention after S1R. (2) Patients with obstruction had significantly higher common filling pressures and decreased systolic ventricular function before intervention. (3) Balloon angioplasty was successful in relieving recurrent arch obstruction. (4) Arch obstruction before S2R did not affect interstage sudden death. (5) Common atrial pressure and increased atrioventricular valve regurgitation remained at time of Fontan in the absence of continued arch obstruction. (6) Recurrent arch obstruction did not affect Fontan morbidity, mortality, or intermediate survival.

Recurrent arch obstruction after S1R for HLHS and other single-ventricle variants has been observed to range from $9 \%$ to $37 \%$. $^{7-14}$ In this series we found an incidence of $23 \%$, with the need for multiple balloon angioplasties also high at $16 \%$. The majority of interventions for recurrent arch obstruction were between S1R and S2R, a vulnerable period for interstage death in this patient population. Despite improved overall survival, it has been suggested that interstage death may be casually related to the occurrence of re-coarctation. ${ }^{9}$ However, although there was not a systematic approach to evaluation for recurrent arch obstruction and limited autopsy data, data from this analysis and our previous evaluations of risk factors for interstage death do not support this association in the current era. ${ }^{19}$

As has been previously documented in several studies, significant, qualitative ventricular systolic dysfunction was common in those patients with the increased afterload of arch obstruction. ${ }^{13,15}$ In addition, the increased afterload may have contributed to the higher incidence of moderate to severe atrioventricular valve regurgitation at the time of re-coarctation. Notably, even in the absence of continued arch obstruction at the time of the Fontan completion, common atrial filling pressures and significant atrioventricular valve regurgitation remained. These findings were in the absence of ongoing increased afterload from arch obstruction and may be related to diastolic dysfunction, ventricular hypertrophy, or other changes that occurred at the time of the recurrent arch obstruction.

Several studies of recurrent arch obstruction have shown that mid- and long-term survivals are affected by re-coarctation without a specific causality. ${ }^{13,20}$ In this series, recurrent arch obstruction did not have an impact on mortality or need for transplantation at a median of 58 months. The primary objective of this study was to investigate the relationship of recurrent arch obstruction and suitability for Fontan completion. We did not find that recurrent arch obstruction, even in patients who required both balloon angioplasty and surgical revision or multiple balloon dilations, had a negative impact on the ability to be palliated through Fontan completion. Its effect on long-term ventricular function was not evaluated in this study but remains of great concern.

\section{Study Limitations}

This study is limited by its retrospective nature and the biases inherent in this type of study design. A nonrandomized patient population was studied, with possible uneven distribution of unrecognized preoperative risk factors between groups. The study population is also a preselected group by virtue of the fact that they had to survive the first 30 days of life. Patients who died before this time with recurrent arch obstruction were not included. Assessment and intervention were not by protocol and may have changed over the course of the study duration. Along these lines, there is an inherent bias in patient selection because those with decreased ventricular systolic function or significant atrioventricular valve regurgitation, in addition to increased filling pressures, were more commonly found in the group that underwent intervention, because we used these hemodynamic indicators as surrogate markers of recurrent arch obstruction. This study might not be powered to evaluate small differences between comparison groups.

\section{CONCLUSIONS}

Recurrent arch obstruction after S1R is a common and significant problem that can result in worse qualitative systolic ventricular function and atrioventricular valve regurgitation. However, with relief and continued monitoring for evidence of re-coarctation by clinical examination, blood pressure gradient changes, change in the degree of ventricular systolic dysfunction, development of atrioventricular valve regurgitation, 2-dimensional and Doppler evidence of arch obstruction by echocardiogram, and subsequent intervention for recurrent arch obstruction, recoarctation does not adversely affect suitability for Fontan completion, need for transplantation, or death in our series. Longer-term follow-up is warranted to evaluate for potential Fontan failure and increased late mortality in this cohort.

\section{References}

1. Bove EL, Lloyd TR. Staged reconstruction for hypoplastic left heart syndrome. Contemporary results. Ann Surg. 1996;224:387-95.

2. Bove EL. Current status of staged reconstruction for hypoplastic left heart syndrome. Pediatr Cardiol. 1998;19:308-15.

3. Mahle WT, Spray TL, Wernovsky G, Gaynor JW, Clark BJ. Survival after reconstructive surgery for hypoplastic left heart syndrome: a 15 year experience from a single institution. Circulation. 2000;102(suppl 3):III36-41.

4. Malec E, Januszewska K, Kolz J, Pajak J. Factors influencing early outcome of Norwood procedure for hypoplastic left heart syndrome. Eur J Cardiothorac Surg. 2000; 18:202-6.

5. Azakie A, Merklinger SL, McCrindle BW, Van Arsdell GS, Lee KJ, Benson LN, et al. Evolving strategies and improving outcomes of the modified Norwood procedure: a 10 year single institution experience. Ann Thorac Surg. 2001;72: 1349-53.

6. Tweddell JS, Hoffman GM, Mussatto KA, Fedderly RT, Berger S, Jaquiss RD, et al. Improved survival of patients undergoing palliation of hypoplastic left heart syndrome: lessons learned from 115 consecutive patients. Circulation. 2002; 106(suppl I):I82-9.

7. Chang AC, Farrell PE Jr, Murdison KA, Baffa JM, Barber G, Norwood WI, et al. Hypoplastic left heart syndrome: hemodynamic and angiographic assessment 
after initial reconstructive surgery and relevance to modified Fontan procedure. J Am Coll Cardiol. 1991;17:1143-9.

8. Chessa M, Dindar A, Vettukattil JJ, Stumper O, Wright JGC, Silove ED, et al. Balloon angioplasty in infants with aortic obstruction after the modified stage I Norwood procedure. Am Heart J. 2000;140:227-31.

9. Zellers TM. Balloon angioplasty for recurrent coarctation of the aorta in patients following staged palliation for hypoplastic left heart syndrome. Am J Cardiol. 1999;84:231-3.

10. Ishino K, Stumper O, De Giovanni JJ, Silove ED, Wright JG, Sethia B, et al. The modified Norwood procedure for hypoplastic left heart syndrome: early to intermediate results of 120 patients with particular reference to aortic arch repair. J Thorac Cardiovasc Surg. 1999;117:920-30.

11. Meliones JN, Snider R, Bove EL, Rosenthal A, Rosen DA. Longitudinal results after first-stage palliation for hypoplastic left heart syndrome. Circulation. 1990;82(Suppl IV):IV151-6.

12. Murdison KA, Baffa JM, Farrell PE, Chang AC, Barber G, Norwood WI, et al. Hypoplastic left heart syndrome. Outcome after initial reconstruction and before modified Fontan procedure. Circulation. 1990;82(Suppl IV): IV199-207.

13. Tworetzky W, McElhinney DB, Burch GH, Teitel DF, Moore P. Balloon arterioplasty of recurrent coarctation after the modified Norwood procedure in infants. Cathet Cardiovasc Interv. 2000;50:54-8.
14. Zeltser I, Menteer J, Gaynor JW, Spray TL, Clark BJ, Kreutzer J, et al. Impact of recoarctation following the Norwood operation on survival in the balloon angioplasty era. J Am Coll Cardiol. 2005;45:1844-8.

15. Larrazabal LA, Tierney ESS, Brown DW, Gauvreau K, Vida VL, Bergersen L, et al. Ventricular function deteriorates with recurrent coarctation in hypoplastic left heart syndrome. Ann Thorac Surg. 2008;86:869-74.

16. Bartram U, Grunenfelder J, Van Praagh R. Causes of death after the modified Norwood procedure: a study of 122 postmortem cases. Ann Thorac Surg. 1997;64:1795-802.

17. Tabbutt S, Dominguez TE, Ravishankar C, Marino BS, Gruber PJ, Wernovsky G, et al. Outcomes after the stage I reconstruction comparing the right ventricular to pulmonary artery conduit with the modified Blalock Taussig shunt. Ann Thorac Surg. 2005;80:1582-90.

18. Ballweg JA, Dominguez TE, Ravishankar C, Gaynor JW, Nicolson SC, Spray TL, et al. A contemporary comparison of the effect of shunt type in hypoplastic left heart syndrome on the hemodynamics and outcome at Fontan completion. J Thorac Cardiovasc Surg. 2010;140:537-44.

19. Hehir DA, Dominguez TE, Ballweg JA, Ravishankar C, Marino BS, Bird GL, et al. Risk factors for interstage death after stage 1 reconstruction of hypoplastic left heart syndrome and variants. J Thorac Cardiovasc Surg. 2008;136:94-9.

20. Fraisse A, Colan SD, Jonas RA, Gauvreau K, Geva T. Accuracy of echocardiography for detection of aortic arch obstruction after stage I Norwood procedure. Am Heart J. 1998;135:230-6. 